University of Wollongong

Research Online

Australian Institute for Innovative Materials -

Papers

Australian Institute for Innovative Materials

$1-1-2013$

\title{
Modulation of the properties of thin ferromagnetic films with an externally applied electric field in ferromagnetic/piezoelectric/ferromagnetic hybrids
}

D Stamopoulos

Demokritos National Centre For Scientific Research

M Zeibekis

Demokritos National Centre For Scientific Research

Shujun Zhang

The Pennsylvania State University, shujun@uow.edu.au

Follow this and additional works at: https://ro.uow.edu.au/aiimpapers

Part of the Engineering Commons, and the Physical Sciences and Mathematics Commons

Research Online is the open access institutional repository for the University of Wollongong. For further information contact the UOW Library: research-pubs@uow.edu.au 


\title{
Modulation of the properties of thin ferromagnetic films with an externally applied electric field in ferromagnetic/piezoelectric/ferromagnetic hybrids
}

\begin{abstract}
In many cases, technological advances are based on artificial low-dimensional structures of heterogeneous constituents, thus called hybrids, that when come together they provide stand-alone entities that exhibit entirely different properties. Such hybrids are nowadays intensively studied since they are attractive for both basic research and oncoming practical applications. Here, we studied hybrids constituted of piezoelectric (PE) and ferromagnetic (FM) components in the form FM/PE/FM, ultimately aiming to provide means for the controlled modulation of the properties of the FM electrodes, originating from the strain imposed to them by the PE mediator when an electric field is applied. The PE component is in single crystal form, $0.71 \mathrm{~Pb}(\mathrm{Mg} 1 / 3 \mathrm{Nb} 2 / 3) 03-0.29 \mathrm{PbTiO3}$ (PMN-PT), while the FM outer layers are Cobalt (Co) in thin film form. Detailed magnetization measurements performed under variation of the electric field applied to PMN-PT demonstrated the efficient modulation of the properties of the Co electrodes at low temperature (coercive field modulation up to $27 \%$ and saturation magnetization absolute modulation up to $4 \%$ at $\mathrm{T}=10 \mathrm{~K}$ for electric field not exceeding $6 \mathrm{kV} / \mathrm{cm}$ ). The modulation degree faints upon increase of the temperature, evidencing that the thermal energy eventually dominates all other relevant energy scales. Candidate mechanisms are discussed for the explanation of these experimental observations. The results presented here demonstrate that commercially available materials can result in quantitatively noticeable effects. Thus, such elemental Co/PMN-PT/Co units can be used as a solid basis for the development of devices. 2013 AIP Publishing LLC.
\end{abstract}

\section{Keywords}

externally, films, ferromagnetic, hybrids, thin, piezoelectric, properties, modulation, field, electric, applied

\section{Disciplines}

Engineering | Physical Sciences and Mathematics

\section{Publication Details}

Stamopoulos, D., Zeibekis, M. \& Zhang, S. J. (2013). Modulation of the properties of thin ferromagnetic films with an externally applied electric field in ferromagnetic/piezoelectric/ferromagnetic hybrids. Journal of Applied Physics, 114 (13), 134309-1-134309-6. 


\section{AIP tambedidyis}

Modulation of the properties of thin ferromagnetic films with an externally applied electric field in ferromagnetic/piezoelectric/ferromagnetic hybrids

D. Stamopoulos, M. Zeibekis, and S. J. Zhang

Citation: Journal of Applied Physics 114, 134309 (2013); doi: 10.1063/1.4824373

View online: http://dx.doi.org/10.1063/1.4824373

View Table of Contents: http://scitation.aip.org/content/aip/journal/jap/114/13?ver=pdfcov

Published by the AIP Publishing

\section{Articles you may be interested in}

Electric-field-induced spin wave generation using multiferroic magnetoelectric cells

Appl. Phys. Lett. 104, 082403 (2014); 10.1063/1.4865916

Manganite-based magnetic tunnel junction with piezoelectric barrier

J. Appl. Phys. 105, 07 C907 (2009); 10.1063/1.3054380

Temperature dependence of coercivity and magnetic reversal in Sm Co $\mathrm{x}$ thin films

J. Appl. Phys. 97, 10F302 (2005); 10.1063/1.1852215

Structure and magnetic properties of electrodeposited, ferromagnetic, group 3-d element films grown onto GaAs (011) substrate

J. Appl. Phys. 93, 7634 (2003); 10.1063/1.1543916

Theory of low-frequency magnetoelectric effects in ferromagnetic-ferroelectric layered composites

J. Appl. Phys. 92, 7681 (2002); 10.1063/1.1522834

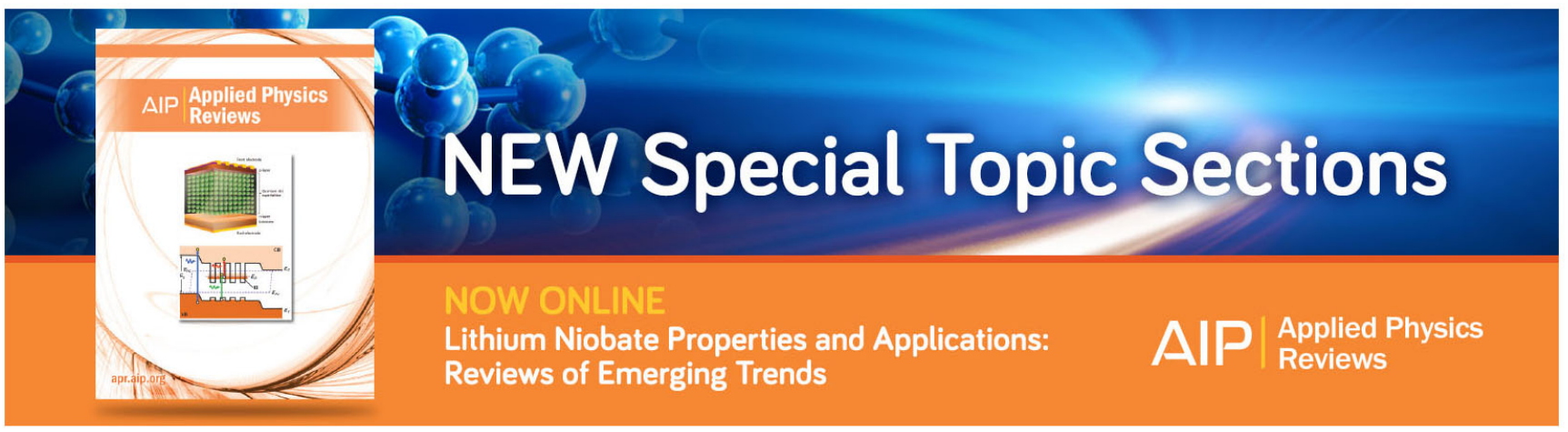




\title{
Modulation of the properties of thin ferromagnetic films with an externally applied electric field in ferromagnetic/piezoelectric/ferromagnetic hybrids
}

\author{
D. Stamopoulos, ${ }^{1, a)}$ M. Zeibekis, ${ }^{1}$ and S. J. Zhang ${ }^{2}$ \\ ${ }^{1}$ Institute of Advanced Materials, Physicochemical Processes, Nanotechnology and Microsystems, \\ "National Center for Scientific Research" "Demokritos," Aghia Paraskevi, Athens 15310, Greece \\ ${ }^{2}$ Materials Research Institute, Pennsylvania State University, University Park, Pennsylvania 16802, USA
}

(Received 2 August 2013; accepted 20 September 2013; published online 4 October 2013)

\begin{abstract}
In many cases, technological advances are based on artificial low-dimensional structures of heterogeneous constituents, thus called hybrids, that when come together they provide stand-alone entities that exhibit entirely different properties. Such hybrids are nowadays intensively studied since they are attractive for both basic research and oncoming practical applications. Here, we studied hybrids constituted of piezoelectric (PE) and ferromagnetic (FM) components in the form FM/PE/FM, ultimately aiming to provide means for the controlled modulation of the properties of the FM electrodes, originating from the strain imposed to them by the PE mediator when an electric field is applied. The PE component is in single crystal form, $0.71 \mathrm{~Pb}\left(\mathrm{Mg}_{1 / 3} \mathrm{Nb}_{2 / 3}\right) \mathrm{O}_{3}-0.29 \mathrm{PbTiO}_{3}$ (PMN-PT), while the FM outer layers are Cobalt $(\mathrm{Co})$ in thin film form. Detailed magnetization measurements performed under variation of the electric field applied to PMN-PT demonstrated the efficient modulation of the properties of the Co electrodes at low temperature (coercive field modulation up to $27 \%$ and saturation magnetization absolute modulation up to $4 \%$ at $\mathrm{T}=10 \mathrm{~K}$ for electric field not exceeding $6 \mathrm{kV} / \mathrm{cm}$ ). The modulation degree faints upon increase of the temperature, evidencing that the thermal energy eventually dominates all other relevant energy scales. Candidate mechanisms are discussed for the explanation of these experimental observations. The results presented here demonstrate that commercially available materials can result in quantitatively noticeable effects. Thus, such elemental Co/PMN-PT/Co units can be used as a solid basis for the development of devices. (C) 2013 AIP Publishing LLC. [http://dx.doi.org/10.1063/1.4824373]
\end{abstract}

\section{INTRODUCTION}

In the recent decades, artificial low-dimensional structures are intensively studied due to the exotic features that they exhibit, making such units attractive for both basic research and practical applications. For instance, the modulation of the properties of a superconductor (SC) by the exchange field (superconducting spin valve effect) and/or stray fields (superconducting magnetoresistance effect) provided by an adjacent ferromagnet (FM) has been studied in great detail both experimentally ${ }^{1-5}$ and theoretically ${ }^{6,7}$ in various versions of SC/FM hybrids. Another example comes from the field of exchange bias where the exchange coupling between a FM and an adjacent antiferromagnet is effective only in the limit of thin films since the cornerstone macroscopic parameter involved in this effect, the so-called exchange bias field, is inversely proportional to the thickness of the FM layer due to the limited penetration of the coupling field inside its interior. ${ }^{8,9}$ In the so-called proximity effect that occurs between a SC and a normal metal (NM), the superconducting order parameter is weakened at the side of the SC layer as it penetrates the NM layer for a few tens of nanometers by the interface. As a consequence, a reduction of the SC critical temperature is observed that is noticeable only when the SC layer is quite thin, since it is inversely

\footnotetext{
a) Author to whom correspondence should be addressed. Electronic mail: densta@ims.demokritos.gr
}

proportional to the square of its thickness. ${ }^{10-12}$ Finally, an interesting class of hybrids that is intensively studied recently is the one composed of FM films adjacent to a piezoelectric (PE) substrate. ${ }^{13-19}$ These hybrids have attracted the interest of researchers, since except for basic science they are promising for numerous devices and sensors, as for instance in magnetic recording for information storage.

Here, we studied hybrids of this latter class that constitute of $\mathrm{PE}$ and $\mathrm{FM}$ materials, namely $0.71 \mathrm{~Pb}\left(\mathrm{Mg}_{1 / 3} \mathrm{Nb}_{2 / 3}\right) \mathrm{O}_{3^{-}}$ $0.29 \mathrm{PbTiO}_{3}$ (PMN-PT) and Cobalt (Co). The PMN-PT component is in single crystal form, while Co comes in thin film form, altogether composing a FM/PE/FM hybrid. In this elemental unit, the Co thin films are employed as electrodes to apply a voltage (that is electric field) to the PMN-PT interlayer. The strain induced to the PMN-PT component is expected to be ultimately delivered to the Co electrodes, thus modulating their magnetic properties. Detailed magnetization measurements performed under controlled variation of the electric field demonstrated a quantitatively noticeable modulation of the properties of the Co electrodes at low temperatures, which faints upon increase of the temperature, a clear sign that the thermal energy eventually dominates all other relevant energy scales. Candidate mechanisms are discussed for the explanation of these experimental observations. Except for basic research, the results presented here demonstrate that commercially available materials that are well studied and are relatively easily prepared can fit together to form hybrids that exhibit quantitatively noticeable effects. Thus, such 
commercial materials can be used as a solid basis for the development of PE/FM-based devices useful in practical applications.

\section{MATERIALS AND METHODS}

\section{A. PMN-PT single crystals}

The relaxor ferroelectric PMN-PT crystals have been grown either by a modified Bridgman method or with the solid-state crystal growth technique $\mathrm{e}^{20-22}$ by using high purity (99.9\%) powders of $\mathrm{Pb}_{3} \mathrm{O}_{4}, \mathrm{MgNb}_{2} \mathrm{O}_{6}$, and $\mathrm{TiO}_{2}$ as starting materials. The specific 0.71PMN-0.29PT single crystals used here have a ferroelectric phase transition at $\mathrm{T}_{\mathrm{RT}}=95^{\circ} \mathrm{C}$ and a Curie phase transition at $\mathrm{T}_{\mathrm{C}}$ $=135^{\circ} \mathrm{C}^{20-22}$ The single crystals were finally cut at rectangular shape with dimensions of $6 \times 5 \times 0.5 \mathrm{~mm}^{3}$, with large face perpendicular to [011] direction and side faces of (100) and $(0-11)$. Thus, upon electric field application, the displacements along the $\mathrm{z}$ direction (that is thickness) and in the xy plane (that is in-plane) are based on the values of $\mathrm{d}_{33}, \mathrm{~d}_{31}$, and $\mathrm{d}_{32}$, being on the order of 800,490 , and $-1200 \mathrm{pC} / \mathrm{N}(\mathrm{pm} / \mathrm{V})$, respectively. After preparation, the crystals were left unpoled.

The as prepared PMN-PT crystals exhibit a relatively rough surface landscape with a mean surface roughness in the range of $400-600 \mathrm{~nm}$. Thus, they were thoroughly polished by using fine sandpaper (silicon-carbide P2500) so that the mean surface roughness was safely reduced below $100 \mathrm{~nm}$, exhibiting typical values in the range of $30-100 \mathrm{~nm}$. The reasons making necessary the reduction of surface roughness are discussed below (see Sec. III). The sandpaper processed PMN-PT crystals are termed polished and have reduced thickness, in respect to the as prepared ones, that ranges between approximately $0.5 \mathrm{~mm}$ (minimally processed crystals) down to $0.3 \mathrm{~mm}$ (extendedly processed ones).

\section{B. Co thin films}

The Co outer layers were RF-sputtered $(30 \mathrm{~W})$ using an Edwards 306A unit [Edwards, Sanborn, NY, USA] at $3 \times 10^{-3}$ Torr of $\operatorname{Ar}(99.999 \%)$ only when a base pressure in the range of $10^{-6}-10^{-7}$ Torr was established upon adequate pumping. The high-quality FM Co outer layers act as electrodes for the application of the voltage (electric field) to the PE crystal, so that the produced strain will be eventually experienced by the Co thin films, as well. The thickness of Co layers investigated here ranges within 30 and $50 \mathrm{~nm}$. In this range, the Co layers are thick to ensure the rather uniform coverage of the surface of the polished PMN-PT crystal (thus, enabling the application of a rather uniform electric field), though staying thin enough to preserve the active layer by the PE/FM interface where any effect is expected to occur.

\section{Crystallographic data}

$\mathrm{X}$-ray diffraction (XRD) spectra were collected with a SIEMENS D500 powder diffractometer using $\mathrm{Cu}-\mathrm{Ka}$ radiation (Bragg-Brentano geometry).

\section{Atomic force microscopy data}

Atomic Force Microscopy (AFM) data were acquired by means of a scanning probe microscope NT-MDT Solver PRO [NT-MDT Co, Moscow, Russia] having a $150 \times 150$ $\times 5 \mu \mathrm{m}^{3}$ xyz-scanner hosted on an active vibration isolation table Halcyonics MOD-1M Plus [Accurion, Goettingen, Germany]. Our measurements were performed in the non-contact scanning mode with NCHR cantilevers that end to silicon nitride tips [Nano and More $\mathrm{GmbH}$, Wetzlar, Germany] having the nominal parameters, spring constant $=42 \mathrm{~N} \mathrm{~m}^{-1}$ and resonance frequency $=320 \mathrm{kHz}$. The optimum imaging results are obtained when the scanning parameters range within: line frequency $=1-2 \mathrm{~Hz}$, area $<30 \times 30 \mu \mathrm{m}^{2}$, and lines per image $=256-512$. The surface roughness (mean surface roughness: $\langle\mathrm{Sa}\rangle=\langle|\mathrm{z}-\langle\mathrm{z}\rangle|\rangle$ ) was calculated by means of the instrument software from images of dimensions $20 \times 20 \mu \mathrm{m}^{2}$.

\section{E. Magnetization data}

Detailed magnetization measurements were performed by using a homemade, hollow sample rod (outer diameter $3 \mathrm{~mm}$, wall thickness $0.25 \mathrm{~mm}$ ) hosting two copper wires (diameter $0.1 \mathrm{~mm}$ ) used to apply the external voltage to the hybrid sample Co/PMN-PT/Co. We stress that the copper wires were adequately thin to minimize heat transfer from the top part of the sample rod (being outside the cryostat, at room temperature) to the cryostat chamber. This detail is very important to be able to maintain temperatures as low as $10 \mathrm{~K}$ and explore the properties of the PE/FM hybrids. A commercial SQUID magnetometer MPMS 5.5 T [Quantum Design, San Diego, CA, USA] was used as the host cryostat for the experiments. In all magnetic measurements presented here, the magnetic field was applied parallel to the hybrid surface. Thus, in this configuration the external magnetic and electric fields were normal. The measuring protocol of the isothermal $\mathrm{m}(\mathrm{H})$ loops was as follows: we start from $\mathrm{T}=300 \mathrm{~K}$ with zero magnetic field $\mathrm{H}$, apply the electric field $\mathrm{E}$, and cool the FM/PE/FM hybrid to the desired temperature (electric-field cooled process). Then, the magnetic field $\mathrm{H}$ is increased while measuring the $\mathrm{m}(\mathrm{H})$ loop. The full temperature range from $10 \mathrm{~K}$ up to $300 \mathrm{~K}$ was investigated.

\section{RESULTS AND DISCUSSION}

Starting with the standard crystallographic characterization of the PMN-PT crystals, Figure 1 shows typical XRD data for a representative single crystal at room temperature. From these data, we see that the PE single crystals are along [110] direction. The respective XRD data for the Co outer layers (not shown) evidence the preparation of high quality polycrystalline thin films.

From the study of relevant artificial structures of heterogeneous constituents, ${ }^{1-12}$ it is well known that any effect occurring by the interface of layered hybrids has only limited range, thus penetrating at a length scale ranging between a few nanometers to a few tens of nanometers at the interior of each constituent layer. Following this guideline, for the FM/PE/FM hybrids studied here, the FM outer layers should 


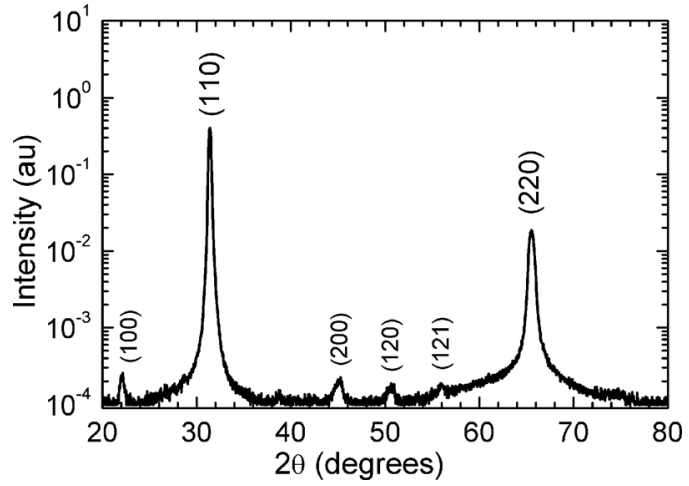

FIG. 1. Representative XRD data for a PMN-PT single crystal.

have the lowest possible thickness since this will ensure that the part of the Co layers affected from the strain provided by the PE crystal will be the maximum. Thus, ideally, the thickness of the Co outer layers cannot exceed a few tens of nanometers (let us say $50 \mathrm{~nm}$ ). An extra reason for working with Co layers with thickness in the range of $30-50 \mathrm{~nm}$ comes from the fact that changes in the magnetic anisotropy of shape origin should be fully effective here; for very thin films (below $30 \mathrm{~nm}$ ), an in-plane magnetic anisotropy dominates, while for sufficiently thick films (above $50 \mathrm{~nm}$ ) an out-of-plane magnetic anisotropy is established. ${ }^{23-25}$

Unfortunately, the requisite of using Co thickness in the range of tens of nanometers meets a strong conflict originating from the morphology of the PE crystals employed here; the as-prepared PMN-PT single crystals have a mean surface roughness in the range of many hundred nanometers as this is evidenced by detailed AFM data (see below). Thus, Co outer layers with thickness of only a few tens of nanometers would probably be ineffective electrodes for voltage delivery since their comparatively small thickness (in respect to the PMN-PT surface roughness) cannot ensure the homogeneous covering of the PMN-PT crystal. To keep the thickness of the Co layers to a minimum and at the same time to deposit relatively homogeneous layers to the PMN-PT crystals, the surface roughness of the latter should be reduced. To that effect, the PMN-PT crystals were thoroughly polished by using fine sandpaper (see Subsection II A) until the mean surface roughness, as assessed by detailed AFM measurements, was safely reduced below $100 \mathrm{~nm}$.

Figures 2(a) and 2(b) show the three-dimensional rendering of representative AFM scans for a PMN-PT single crystal, prior and after polishing, respectively. Both scans refer to an area of $20 \times 20 \mu \mathrm{m}^{2}$ while, as expected, the scale along the $\mathrm{z}$ axis is quite different. The specific areas shown here exhibit $\langle\mathrm{Sa}\rangle=433 \mathrm{~nm}$, prior (Figure 2(a)) and $\langle\mathrm{Sa}\rangle=37 \mathrm{~nm}$, after (Figure 2(b)) polishing. The mean surface roughness $\langle\mathrm{Sa}\rangle$ of the specific single crystal, after investigation of 10 different areas for meeting adequate statistics, is $\langle\mathrm{Sa}\rangle=517 \pm 94 \mathrm{~nm}$, prior and $\langle\mathrm{Sa}\rangle=82 \pm 32 \mathrm{~nm}$, after polishing. When the surface roughness of the PMN-PT crystals was adequately reduced, Co layers with thickness in the range of a few tens of nanometers were deposited. The magnetic properties were thoroughly studied for electric field values $\mathrm{E}=0-6 \mathrm{kV} / \mathrm{cm}$ and temperatures $\mathrm{T}=10-300 \mathrm{~K}$. (a)

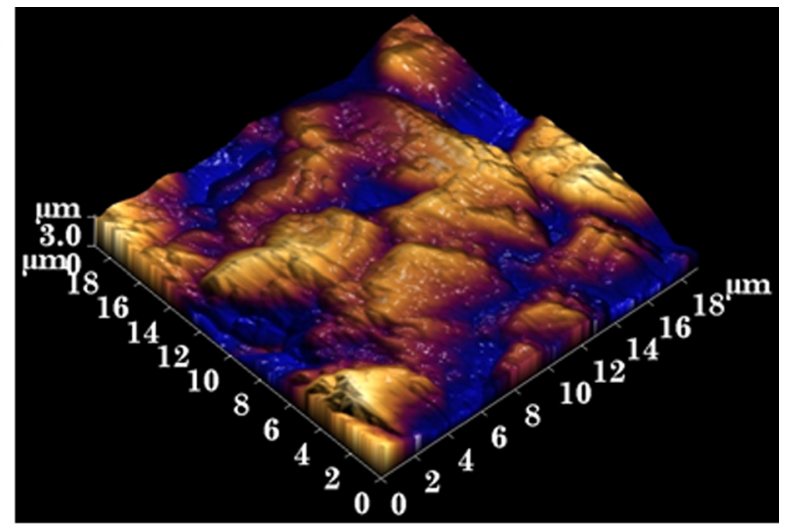

(b)

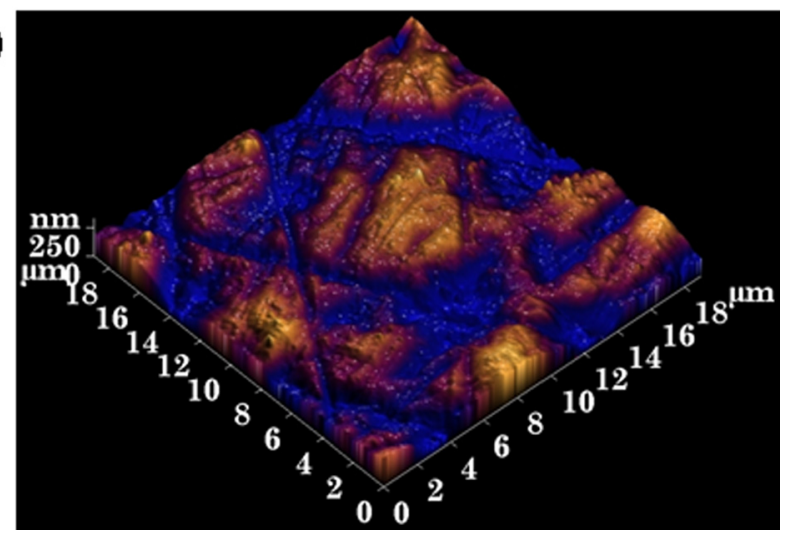

FIG. 2. Typical AFM image $\left(20 \times 20 \mu \mathrm{m}^{2}\right)$, in three-dimensional rendering, of a PMN-PT single crystal (a) prior and (b) after polishing. The mean surface roughness $\langle\mathrm{Sa}\rangle$ of the specific areas is (a) $433 \mathrm{~nm}$ and (b) $37 \mathrm{~nm}$, prior and after polishing, respectively. Notice the different scale along the $\mathrm{z}$ axis between (a) and (b).

In Figures 3(a)-3(d), we show representative $\mathrm{m}(\mathrm{H})$ loops obtained in an $\mathrm{Co}(30 \mathrm{~nm}) / \mathrm{PMN}-\mathrm{PT}(0.5 \mathrm{~mm}) / \mathrm{Co}(30 \mathrm{~nm})$ hybrid for various values of the applied electric field, $\mathrm{E}=0$, 4 , and $6 \mathrm{kV} / \mathrm{cm}$. Two characteristic temperatures are presented, the lowest $\mathrm{T}=10 \mathrm{~K}$ (panels (a) and (c)) and the highest $\mathrm{T}=300 \mathrm{~K}$ (panels (b) and (d)) studied in this work. These data document clearly the modulation of the coercive field, $\mathrm{H}_{\mathrm{c}}$ (main panels) and of the saturation magnetization, $\mathrm{m}_{\mathrm{sat}}$ (insets) of the outer FM electrodes upon application of the electric field, E. Qualitatively, the modulation of $\mathrm{H}_{\mathrm{c}}$ upon $\mathrm{E}$ is non-monotonic, while that of $\mathrm{m}_{\mathrm{sat}}$ is monotonic. Quantitatively, at low temperature $\mathrm{T}=10 \mathrm{~K}$, the coercive field $\mathrm{H}_{\mathrm{c}}$ exhibits a noticeable percentage modulation, as defined by equation $\left[\left(\mathrm{H}_{\mathrm{c}}^{\mathrm{E} 2}-\mathrm{H}_{\mathrm{c}}^{\mathrm{E} 1}\right) / \mathrm{H}_{\mathrm{c}}^{\mathrm{E} 1}\right] 100 \%$, of order $27 \%$ between $\mathrm{E}_{2}=4 \mathrm{kV} / \mathrm{cm}$ and $\mathrm{E}_{1}=6 \mathrm{kV} / \mathrm{cm}$ (where $\mathrm{H}_{\mathrm{c}}{ }^{\mathrm{E}}=\left(\mathrm{H}_{\mathrm{c}+}{ }^{\mathrm{E}}+\left|\mathrm{H}_{\mathrm{c}-}{ }^{\mathrm{E}}\right|\right) / 2$ is the effective coercivity recorded at electric field $\mathrm{E}$ ). At the same temperature $\mathrm{T}=10 \mathrm{~K}$, the saturation magnetization $\mathrm{m}_{\mathrm{sat}}$ exhibits a lower percentage absolute modulation, as defined by equation $\left[\left(\mathrm{m}_{\mathrm{sat}}^{\mathrm{E} 2}-\mathrm{m}_{\mathrm{sat}}^{\mathrm{E} 1}\right) /\right.$ $\left.\mathrm{m}_{\mathrm{sat}}^{\mathrm{E} 1}\right] 100 \%$, of order $4 \%$ between $\mathrm{E}_{2}=6 \mathrm{kV} / \mathrm{cm}$ and $\mathrm{E}_{1}$ $=0 \mathrm{kV} / \mathrm{cm}$ (where $\mathrm{m}_{\text {sat }} \mathrm{E}$ is the saturation magnetization recorded at electric field $\mathrm{E}$ ).

These data are quantitatively summarized in Figures 4(a) and 4(b) wherein the modulation of the coercive field, $\mathrm{H}_{\mathrm{c}}$ and saturation magnetization, $\mathrm{m}_{\mathrm{sat}}$ are shown upon application of electric field, $\mathrm{E}$ at the lowest $\mathrm{T}=10 \mathrm{~K}$ and highest $\mathrm{T}=300 \mathrm{~K}$ temperature, respectively. For the sake 
(a)

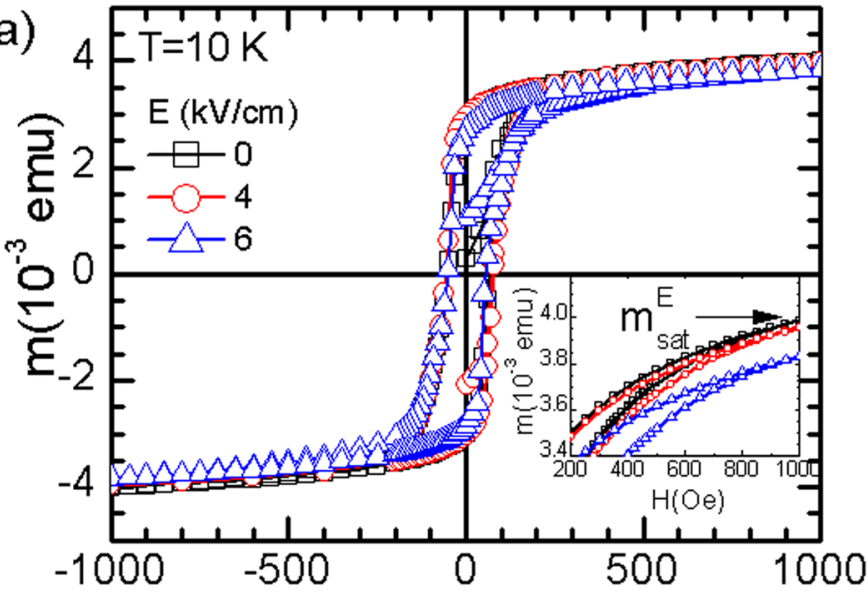

(c)

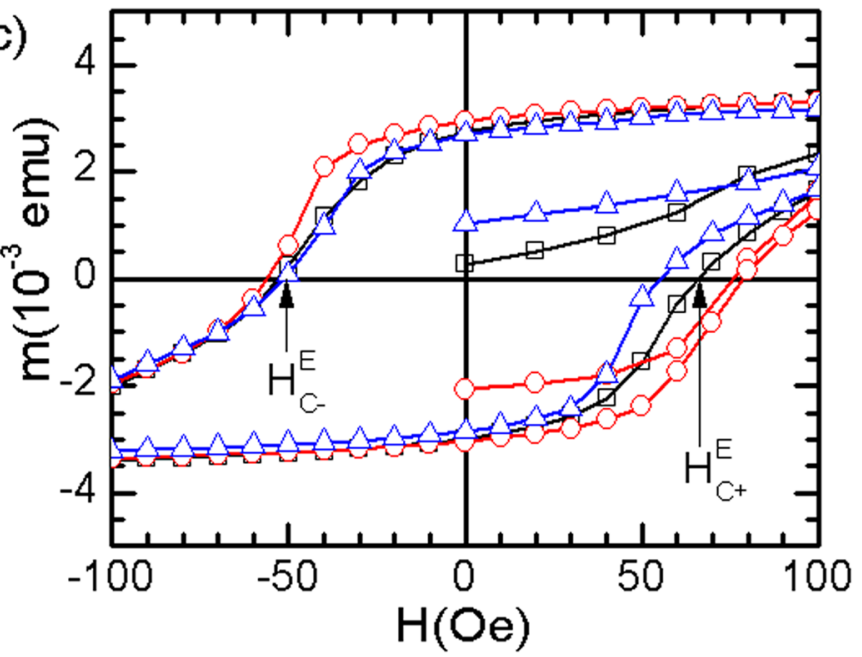

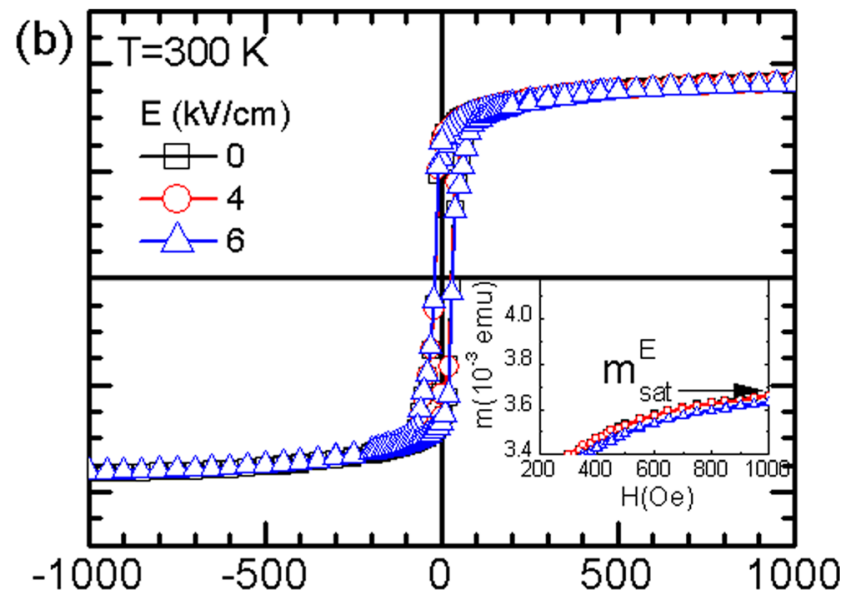

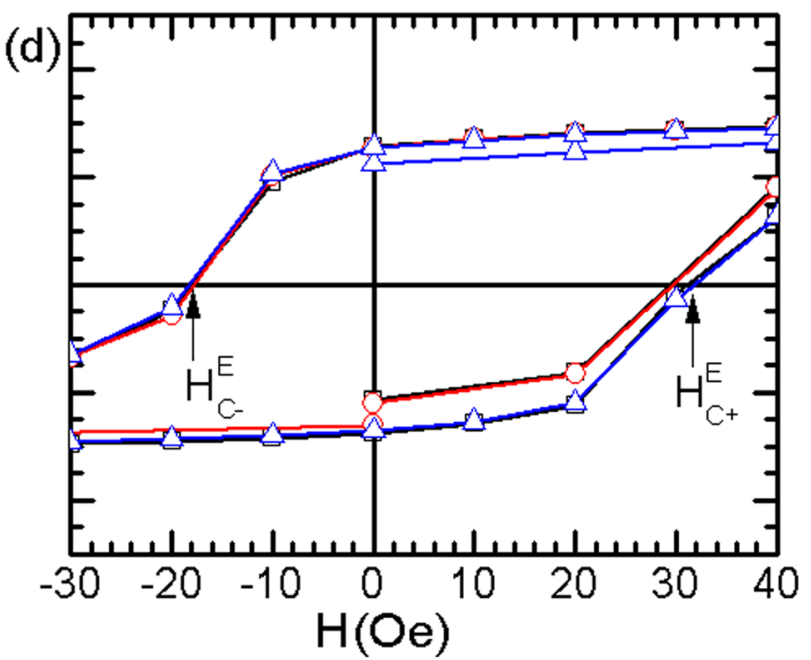

FIG. 3. Isothermal $\mathrm{m}(\mathrm{H})$ loops for various electric fields, E values at (a), (c) $\mathrm{T}=10 \mathrm{~K}$ and (b), (d) $\mathrm{T}=300 \mathrm{~K}$ for (a), (b) high and (c), (d) low magnetic fields. The insets in (a) and (b) focus on the saturation magnetization at the maximum applied magnetic field.

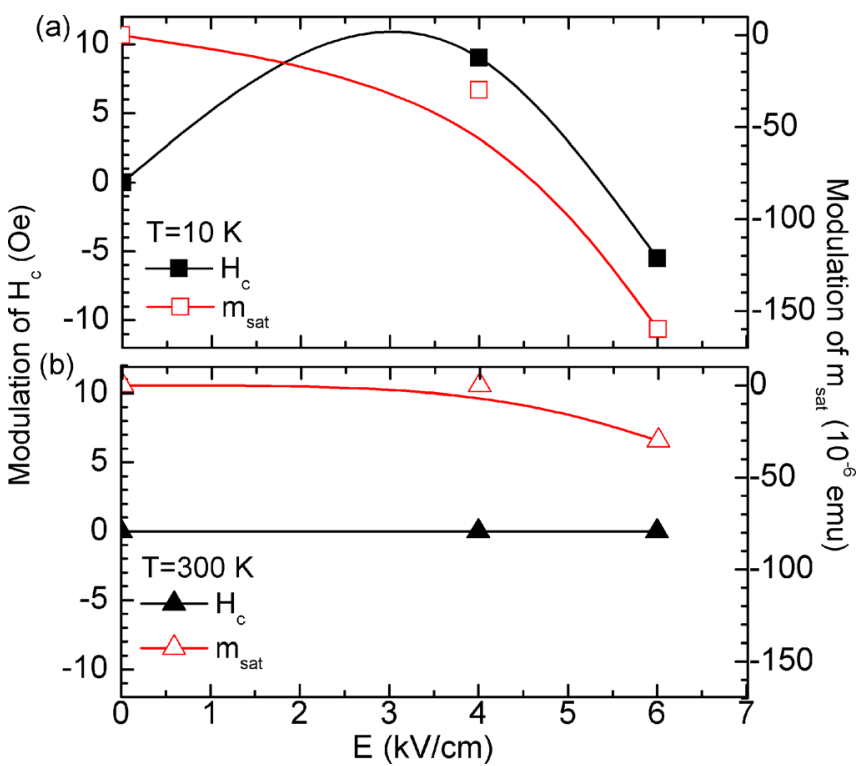

FIG. 4. Modulation of the coercive field, $\mathrm{H}_{\mathrm{c}}$ (solid symbols) and saturation magnetization, $\mathrm{m}_{\mathrm{sat}}$ (open symbols) upon application of electric field, $\mathrm{E}$ at (a) $\mathrm{T}=10 \mathrm{~K}$ and (b) $\mathrm{T}=300 \mathrm{~K}$. Notice that in these plots the same scale is used for direct quantitative comparison of the data. of direct quantitative comparison, the respective plots are presented at the same scale. From these data, it becomes evident that the coercive field $\mathrm{H}_{\mathrm{c}}$ is strongly modulated upon electric field application at low temperature $\mathrm{T}=10 \mathrm{~K}$, as shown in Figure 4(a). However, we observed that above $\mathrm{T}=150 \mathrm{~K}$ the coercive field $\mathrm{H}_{\mathrm{c}}$ practically becomes insensitive to electric field application, as shown in Figure 4(b) for $\mathrm{T}=300 \mathrm{~K}$. Regarding the saturation magnetization $\mathrm{m}_{\text {sat }}$, we observed that it is strongly affected by the applied electric field in a much wider temperature regime. Even at room temperature $\mathrm{T}=300 \mathrm{~K}, \mathrm{~m}_{\text {sat }}$ decreases for approximately $30 \times 10^{-6}$ emu $(-0.8 \%)$ when an electric field $\mathrm{E}=6 \mathrm{kV} / \mathrm{cm}$ is applied. The respective decrease at low temperature $\mathrm{T}=10 \mathrm{~K}$ for the same electric field value is $160 \times 10^{-6}$ emu $(-4.0 \%)$.

Regarding the mechanisms that motivate and/or promote the observed effects, we propose that the strain imposed to the FM layers by the PMN-PT crystal can probably have a two-fold action on two basic parameters defining the magnetic properties: (i) the pinning of domain walls by surface/interface roughness and/or "bulk" static disorder" $26-29$ and (ii) the magnetic anisotropy whether of crystalline or shape origin. ${ }^{23-25}$ 
Referring to the coercive field $\mathrm{H}_{\mathrm{c}}$, we believe that the observed modulation is motivated by two candidate mechanisms: (a) by the pinning of already existing domain walls that can be effectively enhanced either by the surface/interface roughness or "bulk" static disorder that are newly introduced by the developed $\operatorname{strain}^{26-29}$ and (b) by the modulation of domain walls population that inevitably should accompany the modulation of magnetic domain size due to the deformation of the FM film thickness motivated by the induced strain (the magnetic domain size $\mathrm{w}$ is proportional to the square root of the film thickness $d$, that is $\left.\mathrm{W} \sim \mathrm{d}^{1 / 2}\right) .23-25$

Referring to the saturation magnetization $\mathrm{m}_{\mathrm{sat}}$, we believe that the observed modulation can be motivated by the change of the magnetic anisotropy due to the change of the shape anisotropy either at the local or global level. For Co it is well known that, except for the magnetocrystalline anisotropy, another mechanism of magnetic anisotropy stems from the dimensional restrictions due to the reduction of the layer thickness (shape anisotropy). Typically, Co films below the critical thickness $30-50 \mathrm{~nm}$ exhibit in-plane magnetization processes, while above $30-50 \mathrm{~nm}$ they attain out-of-plane magnetic configuration. ${ }^{23,24}$ Thus, by working in the "grey area" of $30-50 \mathrm{~nm}$ where the magnetic shape anisotropy of the FM layers is not robust we are probably able to modulate it due to the strain introduced by the PE crystal that can induce shape deformation at the local and/or global level. Modulation of the magnetic anisotropy from in-plane to out-of-plane can result in changes in the saturation magnetization that is measured along the external magnetic field (applied in-plane). This mechanism can also motivate the modulation of the coercive field, $\mathrm{H}_{\mathrm{c}}$ discussed above.

Though of different degree, the response of both coercive field, $\mathrm{H}_{\mathrm{c}}$ and saturation magnetization, $\mathrm{m}_{\mathrm{sat}}$ upon electric field application decreases upon temperature increase. We believe that the similar behavior of $\mathrm{H}_{\mathrm{c}}$ and $\mathrm{m}_{\text {sat }}$ has a common origin: the thermal energy $\mathrm{k}_{\beta} \mathrm{T}$ that progressively counterbalances and ultimately dominates all other relative energy scales (for instance, pinning energy of domain walls) as temperature increases. For room-temperature applications, this is a disadvantage that in future studies on PE/FM hybrids should be rectified either by exploring more complex geometries or by employing other materials. Finally, we have to note that the almost negligible modulation of both coercive field, $\mathrm{H}_{\mathrm{c}}$ and saturation magnetization, $\mathrm{m}_{\mathrm{sat}}$ observed at room temperature is probably due to the fact that the PMN-PT crystal was left unpoled after production. The maximum electric field applied in our experiments was $6 \mathrm{kV} / \mathrm{cm}$ that is probably not enough to induce strong strain to the PMN-PT crystal. The modulation of the magnetic properties of the Co outer layers would probably be more intense if formerly poled PMN-PT crystals were used. This issue could be investigated in a future work.

\section{CONCLUSIONS}

Here, we investigated PE/FM hybrids in the specific form $\mathrm{FM} / \mathrm{PE} / \mathrm{FM}$ that were based on materials that are well studied and easily prepared, namely PMN-PT in single crystal form and $\mathrm{Co}$ in thin-film form. Magnetization measurements performed under variation of the electric field applied to the PMN-PT crystal through the Co electrodes demonstrated the efficient modulation of coercivity (up to 27\%) and saturation magnetization (up to 4\%) of the employed Co outer layers especially in the low temperature regime $(\mathrm{T}=10 \mathrm{~K})$. Candidate mechanisms that are involved in the modulation of coercivity and saturation magnetization in relevant PE/FM hybrids were discussed. Our data evidenced that the modulation degree faints as temperature progressively increases, a clear proof that the thermal energy ultimately dominates all other relevant energy scales. The present report can provide guidance for future studies on controlling the properties of conventional FM thin films through a PE substrate.

\section{ACKNOWLEDGMENTS}

One of the authors, M Zeibekis, acknowledges the "A.G. Leventis Foundation" for financial support through a scholarship.

${ }^{1}$ D. Stamopoulos, E. Aristomenopoulou, E. Manios, and D. Niarchos, J. Supercond. Novel Magn. 26, 1931 (2013).

${ }^{2}$ A. A. Armenio, C. Cirillo, G. Iannon, S. L. Prischepa, and C. Attanasio, Phys. Rev. B 76, 024515 (2007).

${ }^{3}$ D. Stamopoulos, E. Manios, and M. Pissas, Phys. Rev. B 75, 184504 (2007).

${ }^{4}$ D. Stamopoulos, E. Manios, and M. Pissas, Supercond. Sci. Technol. 20, 1205 (2007).

${ }^{5}$ C. Visani, V. Peña, J. Garcia-Barriocanal, D. Arias, Z. Sefriouri, C. Leon, J. Santamaria, N. M. Nemes, M. Garcia-Hernandez, J. L. Martinez, S. G. E. te Velthuis, and A. Hoffman, Phys. Rev. B 75, 054501 (2007).

${ }^{6}$ L. R. Tagirov, Phys. Rev. Lett. 83, 2058 (1999).

${ }^{7}$ A. I. Buzdin, V. Vedyayev, and N. V. Ryzhanova, Europhys. Lett. 48, 686 (1999).

${ }^{8}$ J. Nogués and I. Schuller, J. Magn. Magn. Mater. 192, 203 (1999).

${ }^{9}$ J. G. Hu, G. J. Jin, and Y. Q. Ma, J. Appl. Phys. 94, 2529 (2003).

${ }^{10}$ R. G. Mints and I. B. Snapiro, Phys. Rev. B 57, 10318 (1998).

${ }^{11}$ G. Palasantzas, Solid State Commun. 112, 97 (1999).

${ }^{12} \mathrm{~V}$. V. Schmidt, The Physics of Superconductors: Introduction to Fundamentals and Applications (Springer-Verlag, Berlin, 1997).

${ }^{13}$ S. Polisetty, W. Echtenkamp, K. Jones, X. He, S. Sahoo, and Ch. Binek, Phys. Rev. B 82, 134419 (2010).

${ }^{14}$ S. Brivio, D. Petti, R. Bertacco, and J. C. Cezar, Appl. Phys. Lett. 98, 092505 (2011).

${ }^{15}$ S. Geprägs, A. Brandlmaier, M. Opel, R. Cross, and S. T. B. Goennenwein, Appl. Phys. Lett. 96, 142509 (2010).

${ }^{16}$ A. Alberca, N. M. Nemes, F. J. Mompean, N. Biskup, A. de Andres, C. Munuera, J. Tornos, C. Leon, A. Hernando, P. Ferrer, G. R. Castro, J. Santamaría, and M. Garcia-Hernandez, Phys. Rev. B 84, 134402 (2011).

${ }^{17}$ T. H. E. Lahtinen, K. J. A. Franke, and S. Dijken, Sci. Rep. 2, 258 (2012).

${ }^{18}$ Z. X. Cheng, X. L. Wang, S. X. Dou, M. Osada, and H. Kimura, Appl. Phys. Lett. 99, 092103 (2011).

${ }^{19}$ S. Rizwan, H. F. Liu, X. F. Han, S. Zhang, Y. G. Zhao, and S. Zhang, SPIN 2, 1250006 (2012).

${ }^{20}$ S. J. Zhang, S. M. Lee, D. H. Kim, H. Y. Lee, and T. R. Shrout, J. Am. Ceram. Soc. 91, 683 (2008).

${ }^{21}$ S. Zhang and F. Li, J. Appl. Phys. 111, 031301 (2012).

${ }^{22}$ J. Luo, W. Hackenberger, S. Zhang, and T. R. Shrout, Proc.-IEEE Ultrason. Symp. 968, 5441884 (2009).

${ }^{23}$ M. Hehn, S. Padovani, K. Ounadjela, and J. P. Bucher, Phys. Rev. B 54, 3428 (1996). 
${ }^{24}$ J. Brandenburg, R. Huhne, L. Schultz, and V. Neu, Phys. Rev. B 79, 054429 (2009).

${ }^{25}$ S. P. Li, A. Samad, W. S. Lew, Y. B. Xu, and J. A. C. Bland, Phys. Rev. B 61, 6871 (2000).

${ }^{26}$ E. Arzt, Acta Mater. 46, 5611 (1998).
${ }^{27}$ Y. P. Zhao, R. M. Gamache, G. C. Wang, T. M. Lu, G. Palasantzas, and J. T. M. Hosson, J. Appl. Phys. 89, 1325 (2001).

${ }^{28}$ M. Li, G. C. Wang, and H. G. Min, J. Appl. Phys. 83, 5313 (1998).

${ }^{29}$ M. Li, Y. P. Zhao, G. C. Wang, and H. G. Min, J. Appl. Phys. 83, 6287 (1998). 